\title{
A COUNTEREXAMPLE IN NONLINEAR BOUNDARY VALUE PROBLEMS
}

J. W. HEIDEL

\begin{abstract}
For the boundary value problem (1.1), (1.2) below where initial value problems of (1.1) are unique and exist on $(a, b)$ it is known that global uniqueness on $(a, b)$ implies global existence on $(a, b)$ if $\beta \delta=0$. It is also known that this is false if $\beta \delta \neq 0$ and $\alpha \delta-\beta \gamma=0$. It is shown here by example that this is also false if $\beta \delta \neq 0$ and $\alpha \delta-\beta \gamma \neq 0$.
\end{abstract}

1. Introduction. This paper is concerned with solutions of the nonlinear differential equation

$$
x^{\prime \prime}=f\left(t, x, x^{\prime}\right)
$$

which satisfy the general linear boundary conditions

$$
\alpha x\left(t_{1}\right)+\beta x^{\prime}\left(t_{1}\right)=r_{1}, \quad \gamma x\left(t_{2}\right)+\delta x^{\prime}\left(t_{2}\right)=r_{2} .
$$

It will be assumed throughout that $f$ is a continuous real valued function on $(a, b) \times R^{2}$ and $a, b, \alpha, \beta, \gamma, \delta, r_{1}$, and $r_{2}$ are real constants such that $\alpha^{2}+\beta^{2}>0$ and $\gamma^{2}+\delta^{2}>0$

We recall the following definitions [2].

Definition 1.1. Problem (1.1), (1.2) with any fixed $f, a, b, \alpha, \beta, \gamma, \delta$ is said to be globally unique if for $t_{1}, t_{2} \in(a, b)$ with $t_{1}<t_{2}$ and for every $r_{1}, r_{2} \in R$ there exists at most one solution of (1.1), (1.2).

Definition 1.2. Problem (1.1), (1.2) with any fixed $f, a, b, \alpha, \beta, \gamma, \delta$ is said to be globally solvable if for every $t_{1}, t_{2} \in(a, b)$ with $t_{1}<t_{2}$ and for every $r_{1}, r_{2} \in R$ there exists at least one solution of (1.1), (1.2).

Lasota and Opial [3] have established the following theorem.

THEOREM 1.1. Suppose that for every point $\left(t_{0}, x_{0}, y_{0}\right) \in(a, b) \times R^{2}$ there exists one and only one solution $x(t)$ of equation (1.1) such that $x\left(t_{0}\right)=x_{0}, x^{\prime}\left(t_{0}\right)=y_{0}$ and it is defined on the whole interval $(a, b)$. If problem (1.1), (1.2) with $\beta \delta=0$ is globally unique then the same problem is globally solvable.

Presented to the Society, November 19, 1971; received by the editors July 2, 1971 and, in revised form, October 5, 1971.

AMS 1969 subject classifications. Primary 3404, 3436.

Key words and phrases. Nonlinear boundary value problems, second order differential equations, global uniqueness, global existence.

(c) American Mathematical Society 1972 
Lasota and Opial [3] have also shown that the theorem need not be true if $\beta \delta \neq 0$. In particular they give an example to show that the theorem is not true for $\alpha=\gamma=-1$ and $\beta=\delta=1$. Note that in this case

$$
D=\operatorname{det}\left(\begin{array}{ll}
\alpha & \beta \\
\gamma & \delta
\end{array}\right)=0
$$

If $\beta \delta \neq 0,(1.2)$ can be written in the form

$$
c x\left(t_{1}\right)+x^{\prime}\left(t_{1}\right)=r_{1}^{\prime}, \quad d x\left(t_{2}\right)+x^{\prime}\left(t_{2}\right)=r_{2}^{\prime} .
$$

The transformation

$$
u=x e^{c t}
$$

transforms (1.1), (1.3) into

$$
\begin{gathered}
u^{\prime \prime}=g\left(t, u, u^{\prime}\right), \\
u^{\prime}\left(t_{1}\right)=r_{1}^{\prime \prime}, \quad(d-c) u\left(t_{2}\right)+u^{\prime}\left(t_{2}\right)=r_{2}^{\prime \prime} .
\end{gathered}
$$

Furthermore this transformation preserves global uniqueness in the sense that (1.1), (1.3) is globally unique if and only if (1.5), (1.6) is globally unique. Thus if $\beta \delta \neq 0$ and $D=0,(1.1),(1.2)$ can be transformed into (1.5) and $u^{\prime}\left(t_{1}\right)=r_{1}^{\prime \prime}, u^{\prime}\left(t_{2}\right)=r_{2}^{\prime \prime}$. Hence the example of Lasota and Opial shows that Theorem 1.1 does not hold, without further conditions on $f$, for any $\alpha, \beta, \gamma, \delta$ with $\beta \delta \neq 0$ and $D=0$.

Lasota [2] has recently raised the question as to whether Theorem 1.1 can hold if $\beta \delta \neq 0$ and $D \neq 0$. It is shown in the next section that the answer to this question is no for any such $\alpha, \beta, \gamma, \delta$.

It should be remarked that Theorem 1.1 was established independently by Jackson [1] under the stronger assumption that $\beta=\delta=0$. In Theorem 1.1 the hypothesis of uniqueness of initial value problems can be removed entirely. The hypotheses of extendability of initial value problems and of global uniqueness of (1.1), (1.2) can be relaxed. For a discussion of these improvements see [4]. Lasota's paper [2] cited above discusses some additional relations between uniqueness and existence and has many examples.

2. An example. The following example and the remark which follows shows that Theorem 1.1 cannot hold if $\beta \delta \neq 0$ and $D \neq 0$. It is motivated by an example of Lasota and Opial [3] but is more complicated.

Denote by $\phi(p, q)$ the solution of the equation $\phi+p \arctan \phi=q$, $p>-1$. Consider the differential equation

$$
x^{\prime \prime}=F^{\prime \prime} \phi\left(1 / F^{\prime}, x^{\prime} / F^{\prime}\right)
$$


on the interval $(a, b)=(-1,2)$ where the function $F$ is determined below. Letting $w=x^{\prime},(2.1)$ becomes $w^{\prime}=F^{\prime \prime} \phi\left(1 / F^{\prime}, w / F^{\prime}\right)$ which has the general solution

$$
w(t)=B F^{\prime}(t)+\arctan B
$$

if $F^{\prime}>0$ on $(-1,2)$ since initial value problems of the $w$ equation are unique and a member of (2.2) passes through each point in $(-1,2) \times R$.

Since $x(t)=\int_{0}^{t} w(s) d s+A$, the boundary conditions (1.3) will be satisfied if and only if (with $r_{i}^{\prime}$ replaced by $r_{i}, i=1,2$ )

$$
\begin{aligned}
& c \int_{0}^{t_{1}} w(s) d s+c A+w\left(t_{1}\right)=r_{1}, \\
& d \int_{0}^{t_{2}} w(s) d s+d A+w\left(t_{2}\right)=r_{2} .
\end{aligned}
$$

For definiteness in the example suppose that $d=1$ and $c>1$. Then (2.3) becomes

$$
c \int_{t_{1}}^{t_{2}} w(s) d s+c w\left(t_{2}\right)-w\left(t_{1}\right)=c r_{2}-r_{1} .
$$

Substituting (2.2) into (2.4) yields

$$
\begin{aligned}
H\left(B, t_{1}, t_{2}\right)= & B\left(c\left[F\left(t_{2}\right)+F^{\prime}\left(t_{2}\right)\right]-\left[c F\left(t_{1}\right)+F^{\prime}\left(t_{1}\right)\right]\right) \\
& +(\arctan B)\left[c\left(t_{2}+1\right)-\left(c t_{1}+1\right)\right] \\
= & c r_{2}-r_{1} .
\end{aligned}
$$

Global uniqueness will hold if $\partial H / \partial B \neq 0$ for all $t_{1}, t_{2}, B$ such that $-1<t_{1}<t_{2}<2$ and $-\infty<B<\infty$. For this it suffices to have

$$
F\left(t_{2}\right)+F^{\prime}\left(t_{2}\right) \geqq F\left(t_{1}\right)+(1 / c) F^{\prime}\left(t_{1}\right)
$$

if $t_{2} \geqq t_{1}$. Furthermore in order that global existence fail, it will suffice to find at least one pair $\left(t_{1}, t_{2}\right)$ such that

$$
F\left(t_{2}\right)+F^{\prime}\left(t_{2}\right)=F\left(t_{1}\right)+(1 / c) F^{\prime}\left(t_{1}\right) .
$$

Thus $F(t)$ is to be determined in such a way that $F^{\prime}(t)>0$ on $(-1,2)$ and that (2.6), (2.7) hold.

On $[-1,0]$ determine $F$ as the solution of $F_{1}+(1 / c) F_{1}^{\prime}=1, F_{1}(-1)=0$. Then $F_{1}(t)=1-e^{-c(t+1)}$. Hence $F_{1}^{\prime}>0$ and $F_{1}(0)=1-e^{-c}<1$.

On $[0,1]$, determine $F$ as the solution of

$$
F_{2}+g(t) F_{2}^{\prime}=1, \quad F_{2}(0)=F_{1}(0)
$$

where $g \in C^{1}[0,1]$ is chosen so that $g(0)=1 / c, g(1)=1,1 / c \leqq g(t) \leqq 1$ for 
$0 \leqq t \leqq 1$, and $g^{\prime}(0)=g^{\prime}(1)=0$. (For example, $g(t)=[(c-1) / 2 c] \cos \pi(t-1)+$ $(c+1) / 2 c$.) By comparing (2.8) with $y+(1 / c) y^{\prime}=1$ it is easily seen that $F_{2}(t)<1$ for $0 \leqq t \leqq 1$ and so $F_{2}^{\prime}(t)>0$.

On $[1,2], F$ is determined as the solution of $F_{3}+F_{3}^{\prime}=1, F_{3}(1)=F_{2}(1)$. Thus $F_{3}(t)=1+\left(F_{3}(1)-1\right) e^{1-t}$. Since $F_{3}(1)<1, F_{3}^{\prime}(t)>0$.

Finally then we have

$$
\begin{aligned}
F(t) & =F_{1}(t), & & t \in[-1,0], \\
& =F_{2}(t), & & t \in[0,1], \\
& =F_{3}(t), & & t \in[1,2] .
\end{aligned}
$$

$F \in C^{2}[-1,2]$ since $g^{\prime}(0)=g^{\prime}(1)=0 ; F^{\prime}(t)>0$ on $[-1,2]$ since $F_{1}^{\prime}, F_{2}^{\prime}, F_{3}^{\prime}$ are all positive.

Verifying that global uniqueness (i.e. condition (2.6)) holds is a matter of checking cases. For example, if $-1<t_{1}<t_{2} \leqq 0$, then $F\left(t_{1}\right)+(1 / c) F^{\prime}\left(t_{1}\right)=$ $1<F\left(t_{2}\right)+F^{\prime}\left(t_{2}\right)$ etc.

Global existence does not hold. For example let $t_{1}=-1 / 2, t_{2}=3 / 2$. Then $F\left(t_{1}\right)+(1 / c) F^{\prime}\left(t_{1}\right)=1=F\left(t_{2}\right)+F^{\prime}\left(t_{2}\right)$. Thus $H(B, 1 / 2,3 / 2)=(2 c-1)$ $\arctan B$ and there is no solution if $r_{1}, r_{2}$ are chosen so that

$$
\left|\left(c r_{2}-r_{1}\right) /(2 c-1)\right| \geqq \pi / 2 .
$$

REMARK. It is clear that a similar example could be constructed with $d=-1$ and $c<-1$. But the discussion of $\S 1$ shows that every problem (1.1), (1.3) can be transformed by $u=x e^{c t}$ to (1.5), (1.6) if $d-c>0$ or by $u=x e^{d t}$ to $(1.5)$, and $(c-d) u\left(t_{1}\right)+u^{\prime}\left(t_{1}\right)=r_{1}^{\prime \prime \prime}, u^{\prime}\left(t_{2}\right)=r_{2}^{\prime \prime \prime}$ if $c-d>0$. In each case global uniqueness is preserved by the transformation. Thus the above example and its analogue $(c<d=-1)$ show that Theorem 1.1 does not hold, without further conditions on $f$, for any $\alpha, \beta, \gamma, \delta$ with $\beta \delta \neq 0$ and $D \neq 0$.

REMARK. A theorem of Waltman [5] shows that global uniqueness of (1.1), (1.2) even with $\beta \delta \neq 0$ will imply global existence of the same problem if $f$ satisfies a further condition (in addition to the extendability of initial value problems). For (1.1), (1.3) this condition is:

$$
(d x+y)^{2}+(d y+f(t, x, y))^{2} \rightarrow \infty \text { as } x^{2}+y^{2} \rightarrow \infty
$$

uniformly in $t \in(a, b)$. It is not hard to see that this condition fails for the above example.

\section{REFERENCES}

1. Lloyd K. Jackson, Subfunctions and second-order ordinary differential inequalities, Advances in Math. 2 (1968), 307-363. MR 37 \#5462.

2. Andrzej Lasota, Boundary value problems for second order differential equations, Lecture Notes in Math., no. 144, Springer-Verlag, Berlin and New York, 1970, pp. 140-152. 
3. A. Lasota and Z. Opial, On the existence and uniqueness of solutions of a boundary value problem for an ordinary second-order differential equation, Colloq. Math. 18 (1967), 1-5. MR 36 \#2871.

4. Keith Schrader and Paul Waltman, An existence theorem for nonlinear boundary value problems, Proc. Amer. Math. Soc. 21 (1969), 653-656. MR 39 \#533.

5. Paul Waltman, Existence and uniqueness of solutions of boundary value problems for two dimensional systems of nonlinear differential equations, Trans. Amer. Math. Soc. 153 (1971), 223-234. MR 42 \#3347.

Department of Mathematics, University of Tennessee, Knoxville, Tennessee 37916 